\title{
Serap Bulut
}

\section{Fekete-Szegö problem for subclasses of analytic functions defined by Komatu integral operator}

Received: 3 January 2012 / Accepted: 12 December 2012 / Published online: 10 January 2013

(C) The Author(s) 2013. This article is published with open access at Springerlink.com

Abstract Using the Komatu integral operator, new subclasses of analytic functions are introduced. For these classes, several Fekete-Szegö type coefficient inequalities are derived.

Mathematics Subject Classification 30C45

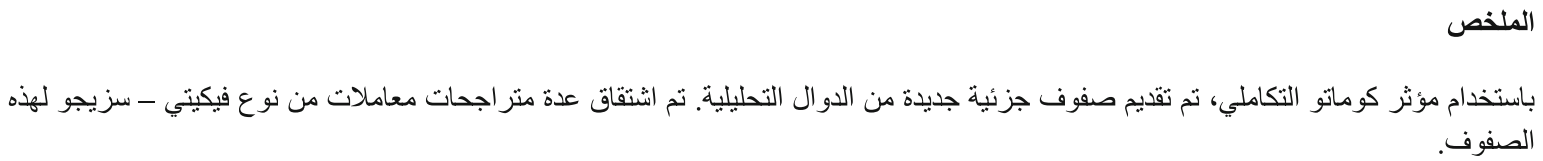

\section{Introduction and definitions}

Let $\mathcal{A}$ denote the class of functions of the form

$$
f(z)=z+a_{2} z^{2}+a_{3} z^{3}+\cdots
$$

which are analytic in the unit disk

$$
\mathbb{U}=\{z \in \mathbb{C}:|z|<1\} .
$$

Also let $\mathcal{S}$ denote the subclass of $\mathcal{A}$ consisting of univalent functions in $\mathbb{U}$.

Fekete and Szegö proved a noticeable result that the estimate

$$
\left|a_{3}-\lambda a_{2}^{2}\right| \leq 1+2 \exp \left(\frac{-2 \lambda}{1-\lambda}\right)
$$

holds for $f \in \mathcal{S}$ and for $0 \leq \lambda \leq 1$. This inequality is sharp for each $\lambda$ (see [8]). The coefficient functional

$$
\phi_{\lambda}(f)=a_{3}-\lambda a_{2}^{2}=\frac{1}{6}\left(f^{\prime \prime \prime}(0)-\frac{3 \lambda}{2}\left(f^{\prime \prime}(0)\right)^{2}\right)
$$

on $f \in \mathcal{A}$ represents various geometric quantities as well as in the sense that this behaves well with respect to the rotation, namely

$$
\phi_{\lambda}\left(\mathrm{e}^{-i \theta} f\left(\mathrm{e}^{i \theta} z\right)\right)=\mathrm{e}^{2 i \theta} \phi_{\lambda}(f) \quad(\theta \in \mathbb{R}) .
$$

S. Bulut $(\varangle)$

Civil Aviation College, Kocaeli University, Arslanbey Campus, 41285 İzmit-Kocaeli, Turkey

E-mail: bulutserap@yahoo.com; serap.bulut@kocaeli.edu.tr 
In fact, other than the simplest case when

$$
\phi_{0}(f)=a_{3},
$$

we have several important ones. For example,

$$
\phi_{1}(f)=a_{3}-a_{2}^{2}
$$

represents $S_{f}(0) / 6$, where $S_{f}$ denotes the Schwarzian derivative

$$
S_{f}(z)=\left(\frac{f^{\prime \prime}(z)}{f^{\prime}(z)}\right)^{\prime}-\frac{1}{2}\left(\frac{f^{\prime \prime}(z)}{f^{\prime}(z)}\right)^{2} .
$$

Moreover, the first two non-trivial coefficients of the $n$-th root transform

$$
\left(f\left(z^{n}\right)\right)^{\frac{1}{n}}=z+c_{n+1} z^{n+1}+c_{2 n+1} z^{2 n+1}+\cdots
$$

of $f$ with the power series (1.1), are written by

$$
c_{n+1}=\frac{a_{2}}{n}
$$

and

$$
c_{2 n+1}=\frac{a_{3}}{n}+\frac{(n-1) a_{2}^{2}}{2 n^{2}}
$$

so that

$$
a_{3}-\lambda a_{2}^{2}=n\left(c_{2 n+1}-\mu c_{n+1}^{2}\right),
$$

where

$$
\mu=\lambda n+\frac{n-1}{2}
$$

Thus, it is quite natural to ask about inequalities for $\phi_{\lambda}$ corresponding to subclasses of $\mathcal{S}$. This is called Fekete-Szegö problem. Actually, many authors have considered this problem for typical classes of univalent functions (see, for instance [1-6, 8,11-13,15,16]).

Recently, Komatu [14] introduced a certain integral operator $L_{a}^{\delta}$ defined by

$$
L_{a}^{\delta} f(z)=\frac{a^{\delta}}{\Gamma(\delta)} \int_{0}^{1} t^{a-2}\left(\log \frac{1}{t}\right)^{\delta-1} f(z t) \mathrm{d} t,
$$

where

$$
a>0 ; \delta \geq 0 ; f(z) \in \mathcal{A} ; z \in \mathbb{U} .
$$

Thus, if $f \in \mathcal{A}$ is of the form (1.1), then it is easily seen from (1.2) that (see [14])

$$
L_{a}^{\delta} f(z)=z+\sum_{n=2}^{\infty}\left(\frac{a}{a+n-1}\right)^{\delta} a_{n} z^{n} .
$$

Using the relation (1.3), it is easy to verify that

$$
z\left(L_{a}^{\delta+1} f(z)\right)^{\prime}=a L_{a}^{\delta} f(z)-(a-1) L_{a}^{\delta+1} f(z)
$$

and

$$
L_{a}^{\delta}\left(z f^{\prime}(z)\right)=z\left(L_{a}^{\delta} f(z)\right)^{\prime}
$$

We note that: 
(i) For $a=1$ and $\delta=k$ ( $k$ is any integer), the multiplier transformation $L_{1}^{k} f(z)=I^{k} f(z)$ was studied by Flett [9] and Salagean [18];

(ii) For $a=1$ and $\delta=-k\left(k \in \mathbb{N}_{0}=\{0,1,2, \ldots\}\right)$, the differential operator $L_{1}^{-k} f(z)=D^{k} f(z)$ was studied by Salagean [18];

(iii) For $a=2$ and $\delta=k$ ( $k$ is any integer), the operator $L_{2}^{k} f(z)=L^{k} f(z)$ was studied by Uralegaddi and Somanatha [19];

(iv) For $a=2$, the multiplier transformation $L_{2}^{\delta} f(z)=I^{\delta} f(z)$ was studied by Jung et al. [10].

Using the operator $L_{a}^{\delta}$, we now introduce the following classes:

Definition 1.1 We say that a function $f \in \mathcal{A}$ is in the class $\mathcal{S}_{a, \delta}(b)$ if

$$
\begin{array}{r}
\operatorname{Re}\left\{1+\frac{1}{b}\left(\frac{z\left(L_{a}^{\delta} f(z)\right)^{\prime}}{L_{a}^{\delta} f(z)}-1\right)\right\}>0 \\
(a>0 ; \delta \geq 0 ; b \in \mathbb{C} \backslash\{0\} ; z \in \mathbb{U}) .
\end{array}
$$

Definition 1.2 We say that a function $f \in \mathcal{A}$ is in the class $\mathcal{C}_{a, \delta}(b)$ if

$$
\begin{aligned}
& \operatorname{Re}\left\{1+\frac{1}{b} \frac{z\left(L_{a}^{\delta} f(z)\right)^{\prime \prime}}{\left(L_{a}^{\delta} f(z)\right)^{\prime}}\right\}>0 \\
& \quad(a>0 ; \delta \geq 0 ; b \in \mathbb{C} \backslash\{0\} ; z \in \mathbb{U}) .
\end{aligned}
$$

Note that

$$
f \in \mathcal{C}_{a, \delta}(b) \Leftrightarrow z f^{\prime} \in \mathcal{S}_{a, \delta}(b) .
$$

In particular, we have starlike and convex function classes,

$$
\mathcal{S}_{a, 0}(1)=\mathcal{S}^{*} \text { and } \mathcal{C}_{a, 0}(1)=\mathcal{C}
$$

respectively.

We denote by $\mathcal{P}$ a class of the analytic functions in $\mathbb{U}$ with

$$
p(0)=1 \text { and } \operatorname{Re}\{p(z)\}>0 .
$$

We shall require the following lemmas.

Lemma 1.3 [7] Let $p \in \mathcal{P}$ with $p(z)=1+c_{1} z+c_{2} z^{2}+\cdots$. Then

$$
\left|c_{n}\right| \leq 2 \quad(n \geq 1)
$$

Lemma 1.4 [17] Let $p \in \mathcal{P}$ with $p(z)=1+c_{1} z+c_{2} z^{2}+\cdots$. Then for any complex number $v$

$$
\left|c_{2}-v c_{1}^{2}\right| \leq 2 \max \{1,|2 v-1|\}
$$

and the result is sharp for the functions given by

$$
p(z)=\frac{1+z^{2}}{1-z^{2}}, \quad p(z)=\frac{1+z}{1-z}
$$

Lemma 1.5 [7] Let $p \in \mathcal{P}$ with $p(z)=1+c_{1} z+c_{2} z^{2}+\cdots$. Then

$$
\left|c_{2}-\frac{1}{2} \mu c_{1}^{2}\right| \leq 2+\frac{1}{2}(|\mu-1|-1)\left|c_{1}\right|^{2} .
$$




\section{Main results}

Theorem 2.1 Let $a>0 ; \delta \geq 0 ; b \in \mathbb{C} \backslash\{0\}$. If $f \in \mathcal{S}_{a, \delta}(b)$, then

$$
\begin{aligned}
& \left|a_{2}\right| \leq 2|b|\left(\frac{a+1}{a}\right)^{\delta}, \\
& \left|a_{3}\right| \leq|b|\left(\frac{a+2}{a}\right)^{\delta} \max \{1,|1+2 b|\},
\end{aligned}
$$

and

$$
\left|a_{3}-\frac{1}{2}\left(\frac{a(a+2)}{(a+1)^{2}}\right)^{\delta} a_{2}^{2}\right| \leq|b|\left(\frac{a+2}{a}\right)^{\delta} .
$$

Proof Denote

$$
L_{a}^{\delta} f(z)=z+A_{2} z^{2}+A_{3} z^{3}+\cdots
$$

Then by (1.3), we can write

$$
A_{2}=\left(\frac{a}{a+1}\right)^{\delta} a_{2}, \quad A_{3}=\left(\frac{a}{a+2}\right)^{\delta} a_{3} .
$$

By the definition of the class $\mathcal{S}_{a, \delta}(b)$, there exists $p \in \mathcal{P}$ such that

$$
\frac{z\left(L_{a}^{\delta} f(z)\right)^{\prime}}{L_{a}^{\delta} f(z)}=1-b+b p(z)
$$

so that

$$
\frac{z\left(1+2 A_{2} z+3 A_{3} z^{2}+\cdots\right)}{z+A_{2} z^{2}+A_{3} z^{3}+\cdots}=1-b+b\left(1+c_{1} z+c_{2} z^{2}+\cdots\right)
$$

which implies the equality

$$
z+2 A_{2} z^{2}+3 A_{3} z^{3}+\cdots=z+\left(A_{2}+b c_{1}\right) z^{2}+\left(A_{3}+b c_{1} A_{2}+b c_{2}\right) z^{3}+\cdots .
$$

Equating the coefficients of both sides, we have

$$
A_{2}=b c_{1}, \quad A_{3}=\frac{b}{2}\left(c_{2}+b c_{1}^{2}\right),
$$

so that, on account of (2.3)

$$
a_{2}=b\left(\frac{a+1}{a}\right)^{\delta} c_{1}, \quad a_{3}=\frac{b}{2}\left(\frac{a+2}{a}\right)^{\delta}\left(c_{2}+b c_{1}^{2}\right) .
$$

Taking into account (2.5) and Lemma 1.3, we obtain

$$
\left|a_{2}\right| \leq 2|b|\left(\frac{a+1}{a}\right)^{\delta},
$$

and Lemma 1.4

$$
\begin{aligned}
\left|a_{3}\right| & =\left|\frac{b}{2}\left(\frac{a+2}{a}\right)^{\delta}\left(c_{2}+b c_{1}^{2}\right)\right| \\
& \leq|b|\left(\frac{a+2}{a}\right)^{\delta} \max \{1,|1+2 b|\} .
\end{aligned}
$$


Moreover, by Lemma 1.3

$$
\begin{aligned}
\left|a_{3}-\frac{1}{2}\left(\frac{a(a+2)}{(a+1)^{2}}\right)^{\delta} a_{2}^{2}\right| & =\left|\frac{b}{2}\left(\frac{a+2}{a}\right)^{\delta}\left(c_{2}+b c_{1}^{2}\right)-\frac{b^{2} c_{1}^{2}}{2}\left(\frac{a(a+2)}{(a+1)^{2}}\right)^{\delta}\left(\frac{a+1}{a}\right)^{2 \delta}\right| \\
& =\left|\frac{b c_{2}}{2}\left(\frac{a+2}{a}\right)^{\delta}\right| \\
& \leq|b|\left(\frac{a+2}{a}\right)^{\delta}
\end{aligned}
$$

as asserted.

Now, we consider functional $\left|a_{3}-\mu a_{2}^{2}\right|$ for complex $\mu$.

Theorem 2.2 Let $a>0 ; \delta \geq 0 ; b \in \mathbb{C} \backslash\{0\}$. If $f \in \mathcal{S}_{a, \delta}(b)$, then for $\mu \in \mathbb{C}$ we have

$$
\left|a_{3}-\mu a_{2}^{2}\right| \leq|b|\left(\frac{a+2}{a}\right)^{\delta} \max \left\{1,\left|1+2 b-4 \mu b\left(\frac{(a+1)^{2}}{a(a+2)}\right)^{\delta}\right|\right\} .
$$

Moreover for each $\mu$, there is a function in $\mathcal{S}_{a, \delta}(b)$ such that equality holds.

Proof Taking into account (2.5) we have

$$
\begin{aligned}
a_{3}-\mu a_{2}^{2} & =\frac{b}{2}\left(\frac{a+2}{a}\right)^{\delta}\left(c_{2}+b c_{1}^{2}\right)-\mu b^{2} c_{1}^{2}\left(\frac{a+1}{a}\right)^{2 \delta} \\
& =\frac{b}{2}\left(\frac{a+2}{a}\right)^{\delta}\left(c_{2}-\tau c_{1}^{2}\right),
\end{aligned}
$$

where

$$
\tau=-b+2 \mu b\left(\frac{(a+1)^{2}}{a(a+2)}\right)^{\delta} .
$$

Then, with the aid of Lemma 1.4, we obtain

$$
\left|a_{3}-\mu a_{2}^{2}\right| \leq|b|\left(\frac{a+2}{a}\right)^{\delta} \max \left\{1,\left|1+2 b-4 \mu b\left(\frac{(a+1)^{2}}{a(a+2)}\right)^{\delta}\right|\right\},
$$

as asserted. An examination of the proof shows that equality is attained for the first case when $c_{1}=0$ and $c_{2}=2$ and the corresponding $f \in \mathcal{S}_{a, \delta}(b)$ is given by

$$
\frac{z\left(L_{a}^{\delta} f(z)\right)^{\prime}}{L_{a}^{\delta} f(z)}=\frac{1+(2 b-1) z^{2}}{1-z^{2}},
$$

and likewise for the second case when $c_{1}=c_{2}=2$ the corresponding $f \in \mathcal{S}_{a, \delta}(b)$ is given by

$$
\frac{z\left(L_{a}^{\delta} f(z)\right)^{\prime}}{L_{a}^{\delta} f(z)}=\frac{1+(2 b-1) z}{1-z}
$$

respectively.

Taking $\delta=0$ and $b=1$ in Theorem 2.2, we have

Corollary 2.3 [12] If $f \in \mathcal{S}^{*}$, then for $\mu \in \mathbb{C}$ we have

$$
\left|a_{3}-\mu a_{2}^{2}\right| \leq \max \{1,|4 \mu-3|\} .
$$

Moreover for each $\mu$, there is a function in $\mathcal{S}^{*}$ such that equality holds. 
We next consider the case when $\mu$ and $b$ are real. Then we have:

Theorem 2.4 Let $a>0 ; \delta \geq 0 ; b>0$. If $f \in \mathcal{S}_{a, \delta}(b)$, then for $\mu \in \mathbb{R}$, we have

$$
\left|a_{3}-\mu a_{2}^{2}\right| \leq \begin{cases}b\left(\frac{a+2}{a}\right)^{\delta}\left[1+2 b-4 \mu b\left(\frac{(a+1)^{2}}{a(a+2)}\right)^{\delta}\right] & \text { if } \mu \leq \frac{1}{2}\left(\frac{a(a+2)}{(a+1)^{2}}\right)^{\delta} \\ b\left(\frac{a+2}{a}\right)^{\delta} & \text { if } \frac{1}{2}\left(\frac{a(a+2)}{(a+1)^{2}}\right)^{\delta} \leq \mu \leq \frac{1+b}{2 b}\left(\frac{a(a+2)}{(a+1)^{2}}\right)^{\delta} \\ b\left(\frac{a+2}{a}\right)^{\delta}\left[-1-2 b+4 \mu b\left(\frac{(a+1)^{2}}{a(a+2)}\right)^{\delta}\right] & \text { if } \mu \geq \frac{1+b}{2 b}\left(\frac{a(a+2)}{(a+1)^{2}}\right)^{\delta}\end{cases}
$$

Moreover for each $\mu$, there is a function in $\mathcal{S}_{a, \delta}(b)$ such that equality holds.

Proof By (2.7), we obtain

$$
a_{3}-\mu a_{2}^{2}=\frac{b}{2}\left(\frac{a+2}{a}\right)^{\delta}\left[c_{2}-\frac{c_{1}^{2}}{2}+\frac{c_{1}^{2}}{2}\left(1+2 b-4 \mu b\left(\frac{(a+1)^{2}}{a(a+2)}\right)\right)\right] .
$$

First, let $\mu \leq \frac{1}{2}\left(\frac{a(a+2)}{(a+1)^{2}}\right)^{\delta}$. In this case, by (2.12), Lemma 1.3 and Lemma 1.5 give

$$
\begin{aligned}
\left|a_{3}-\mu a_{2}^{2}\right| & \leq \frac{b}{2}\left(\frac{a+2}{a}\right)^{\delta}\left[2-\frac{\left|c_{1}\right|^{2}}{2}+\frac{\left|c_{1}\right|^{2}}{2}\left(1+2 b-4 \mu b\left(\frac{(a+1)^{2}}{a(a+2)}\right)\right)\right] \\
& \leq b\left(\frac{a+2}{a}\right)^{\delta}\left[1+2 b-4 \mu b\left(\frac{(a+1)^{2}}{a(a+2)}\right)^{\delta}\right] .
\end{aligned}
$$

Now let $\frac{1}{2}\left(\frac{a(a+2)}{(a+1)^{2}}\right)^{\delta} \leq \mu \leq \frac{1+b}{2 b}\left(\frac{a(a+2)}{(a+1)^{2}}\right)^{\delta}$. Then, using the above calculations, we get

$$
\left|a_{3}-\mu a_{2}^{2}\right| \leq b\left(\frac{a+2}{a}\right)^{\delta}
$$

Finally, if $\mu \geq \frac{1+b}{2 b}\left(\frac{a(a+2)}{(a+1)^{2}}\right)^{\delta}$, then we obtain

$$
\begin{aligned}
\left|a_{3}-\mu a_{2}^{2}\right| & \leq \frac{b}{2}\left(\frac{a+2}{a}\right)^{\delta}\left[2-\frac{\left|c_{1}\right|^{2}}{2}+\frac{\left|c_{1}\right|^{2}}{2}\left(-1-2 b+4 \mu b\left(\frac{(a+1)^{2}}{a(a+2)}\right)^{\delta}\right)\right] \\
& \leq \frac{b}{2}\left(\frac{a+2}{a}\right)^{\delta}\left[2+\frac{\left|c_{1}\right|^{2}}{2}\left(-2-2 b+4 \mu b\left(\frac{(a+1)^{2}}{a(a+2)}\right)^{\delta}\right)\right] \\
& \leq b\left(\frac{a+2}{a}\right)^{\delta}\left[-1-2 b+4 \mu b\left(\frac{(a+1)^{2}}{a(a+2)}\right)^{\delta}\right] .
\end{aligned}
$$

Equality is attained for the second case on choosing $c_{1}=0, c_{2}=2$ in (2.9) and in (2.10) $c_{1}=c_{2}=2 ; c_{1}=$ $2 i, c_{2}=-2$ for the first and third case, respectively. Thus the proof is complete.

Using the relation (1.6), we easily obtain bounds of coefficients and a solution of the Fekete-Szegö problem in $\mathcal{C}_{a, \delta}(b)$.

Theorem 2.5 Let $a>0 ; \delta \geq 0 ; b \in \mathbb{C} \backslash\{0\}$. If $f \in \mathcal{C}_{a, \delta}(b)$, then

$$
\begin{aligned}
& \left|a_{2}\right| \leq|b|\left(\frac{a+1}{a}\right)^{\delta} \\
& \left|a_{3}\right| \leq \frac{|b|}{3}\left(\frac{a+2}{a}\right)^{\delta} \max \{1,|1+2 b|\},
\end{aligned}
$$

and

$$
\left|a_{3}-\frac{2}{3}\left(\frac{a(a+2)}{(a+1)^{2}}\right)^{\delta} a_{2}^{2}\right| \leq \frac{|b|}{3}\left(\frac{a+2}{a}\right)^{\delta}
$$

Reasoning in the same line as in the proof of Theorem 2.2 we obtain 
Theorem 2.6 Let $a>0 ; \delta \geq 0 ; b \in \mathbb{C} \backslash\{0\}$. If $f \in \mathcal{C}_{a, \delta}(b)$, then for $\mu \in \mathbb{C}$ we have

$$
\left|a_{3}-\mu a_{2}^{2}\right| \leq \frac{|b|}{3}\left(\frac{a+2}{a}\right)^{\delta} \max \left\{1,\left|1+2 b-3 \mu b\left(\frac{(a+1)^{2}}{a(a+2)}\right)^{\delta}\right|\right\} \text {. }
$$

Moreover for each $\mu$, there is a function in $\mathcal{C}_{a, \delta}(b)$ such that equality holds.

Taking $\delta=0$ and $b=1$ in Theorem 2.6, we have

Corollary 2.7 [12] If $f \in \mathcal{C}$, then for $\mu \in \mathbb{C}$ we have

$$
\left|a_{3}-\mu a_{2}^{2}\right| \leq \max \left\{\frac{1}{3},|\mu-1|\right\}
$$

Moreover for each $\mu$, there is a function in $\mathcal{C}$ such that equality holds.

Open Access This article is distributed under the terms of the Creative Commons Attribution License which permits any use, distribution, and reproduction in any medium, provided the original author(s) and the source are credited.

\section{References}

1. Abdel-Gawad, H.R.; Thomas, D.K.: The Fekete-Szegö problem for strongly close-to-convex functions. Proc. Am. Math. Soc. 114, 345-349 (1992)

2. Al-Amiri, H.S.: Certain generalization of prestarlike functions. J. Aust. Math. Soc. 28, 325-334 (1979)

3. Choi, J.H.; Kim, Y.Ch.; Sugawa, T.: A general approach to the Fekete-Szegö problem. J. Math. Soc. Jpn. 59(3), 707-727 (2007)

4. Chonweerayoot, A.; Thomas D.K.; Upakarnitikaset, W.: On the Fekete-Szegö theorem for close-to-convex functions. Publ. Inst. Math. (Beograd) (N.S.) 66, 18-26 (1992)

5. Darus, M.; Thomas, D.K.: On the Fekete-Szegö theorem for close-to-convex functions. Math. Jpn. 44, 507-511 (1996)

6. Darus, M.; Thomas, D.K.: On the Fekete-Szegö theorem for close-to-convex functions. Math. Jpn. 47, 125-132 (1998)

7. Duren, P.L.: Univalent Functions, Grundlehren der Mathematics. Wissenschaften, Bd., p. 259. Springer, New York (1983)

8. Fekete, M.; Szegö, G.: Eine bemerkung über ungerade schlichte funktionen. J. Lond. Math. Soc. 8, 85-89 (1933)

9. Flett, T.M.: The dual of an inequality of Hardy and Littlewood and some related inequalities. J. Math. Anal. Appl. 38, $746-765(1972)$

10. Jurg, I.B.; Kim, Y.C.; Srivastava, H.M.: The Hardy space of analytic functions associated with certain one-parameter families of integral operators. J. Math. Anal. Appl. 176, 138-147 (1993)

11. Kanas, S.; Lecko, A.: On the Fekete-Szegö problem and the domain convexity for a certain class of univalent functions. Folia Sci. Univ. Tech. Resov. 73, 49-58 (1990)

12. Keogh, F.R.; Merkes, E.P.: A coefficient inequality for certain classes of analytic functions. Proc. Am. Math. Soc. 20, 8-12 (1969)

13. Koepf, W.: On the Fekete-Szegö problem for close-to-convex functions. Proc. Am. Math. Soc. 101, 89-95 (1987)

14. Komatu, Y.: On analytic prolongation of a family of operators. Math. (Cluj) 32 (55)(2), 141-145 (1990)

15. London, R.R.: Fekete-Szegö inequalities for close-to-convex functions. Proc. Am. Math. Soc. 117, 947-950 (1993)

16. Ma, W.; Minda, D.: A unified treatment of some special classes of univalent functions. In: Li, Z.; Ren, F.; Yang, L.; Zhang, S. (eds.) Proceeding of Conference on Complex Analysis, International Press, pp. 157-169 (1994)

17. Ravichandran, V.; Gangadharan, A.; Darus, M.: Fekete-Szegö inequality for certain class of Bazilevic functions. Far East J. Math. Sci. 15, 171-180 (2004)

18. Salagean, G.S.: Subclasses of univalent functions. In: Lecture Notes in Mathematics, vol. 1013, pp. 362-372. Springer, Berlin (1983)

19. Uralegaddi, B.A.; Somanatha, C.: Certain classes of univalent functions. In: Srivastava, H.M.; Owa, S. (eds.) Current Topics in Analytic Function Theory, pp. 371-374. World Scientific Publishing Company, Singapore (1922) 